\title{
Survey Paper on Fractal Image Compression using Block Truncation Coding Technique
}

\author{
Anshu Agrawal \\ M. Tech. Scholar \\ Department of Computer Science and Engineering \\ Bansal Institute of Research and Technology \\ Bhopal
}

\author{
Pushpraj Singh Chauhan \\ Professor, Head of Department \\ Department of Computer Science and Engineering \\ Bansal Institute of Research and Technology \\ Bhopal
}

\begin{abstract}
Text and image data are important elements for information processing almost in all the computer applications. Uncompressed image or text data require high transmission bandwidth and significant storage capacity. Designing an efficient compression scheme is more critical with the recent growth of computer applications.Modern applications, in addition to high compression ratio, also demand for efficient encoding and decoding processes, so that computational constraint of many real-time applications is satisfied.Two generally utilized spatial area pressure strategies are block truncation coding (BTC) and vector quantization (VQ). BTC strategy brings about great quality picture with high piece rate, while the VQ is notable for low piece rate yet creates low quality pictures.In further work of this paper is multi-level BTC includes BTC algorithm as well as vector quantization method for purpose of multi-leveltechnique for gray and color image.
\end{abstract}

\section{Keywords}

Block Truncation Code (BTC), PSNR, Block Size, Compression Ratio

\section{INTRODUCTION}

The rising multimedia technology and growth of GUI based software have made digital image data an inherent part of modern life. When a 2-D light intensity function is sampled and quantized to create a digital image, the amount of data generated may be large in volume that it results in tremendous storage, processing and communication requirements. Therefore, the theory of data compression becomes more and more important for reducing the data redundancy to save more hardware space and transmission bandwidth.

In software engineering and data hypothesis, information pressure is the way toward encoding data utilizing less number of bits or some other data bearing units. Pressure is valuable as it decreases the utilization of costly assets, for example, hard circle space or transmission data transmission [1] [2]. BTC is a straightforward and quick lossy pressure system for dim scale pictures. The fundamental thought of BTC [3] is to perform minute saving quantization for squares of pixels. The info picture is separated into non-covering pieces of pixels of sizes $4 \times 4,8 \times 8$ et cetera. Mean and standard deviation of the blocks are calculated. Mean is considered as the threshold and reconstruction values are determined using mean and standard deviation.

Then a bitmap of the block is derived based on the value of the threshold which is the compressed or encoded image. Using the reconstruction values and the bitmap the reconstructed image is generated by the decoder. Thus in the encoding process, BTC produces a bitmap, mean and standard deviation for each block. It gives a compression ratio of 4 and bit rate of
2 bits per pixel when a $4 \times 4$ block is considered. This method provides a good compression without much degradation on the reconstructed image. But it shows some artifacts like staircase effects or raggedness near the edges. Due to its simplicity and easy implementation, BTC has gained wide interest in its further development and application for image compression.

To improve the quality of the reconstructed image and for the better compression efficiency several variants of BTC have been developed during the last many years. Supreme Moment Block Truncation Coding (AMBTC) [4] jelly the higher mean and lower mean of each piece and utilize this amount to quantize yield. AMBTC gives preferred picture quality over picture pressure utilizing BTC. In addition, the AMBTC is very quicker contrasted with BTC. The algorithm is computationally faster because it involves simple analytical formulae to compute the parameters of the edge feature in an image block. Reconstructed images are of good quality in accordance with human perceptual experience. The algorithm represents the image in terms of its binary edge map, mean information, and the intensity information on both sides of the edges.

\section{LITERATURE SURVEY}

Sunwoong Kim et al. [1], Frame memory compression is a widely used image compression technique that aims to reduce the size of the frame memory in display panels such as those containing LCD and OLED technologies. Late LCD boards utilize the RGBW shading area to supplant the customary RGB space keeping in mind the end goal to improve the shine of LCD boards with the expansion of a white segment. The extra part expands the extent of the casing memory yet requires a forceful pressure calculation. This paper proposes a novel pressure calculation for RGBW parts that enhances the proficiency of piece truncation coding (BTC), which is generally utilized for LCD overdrive.

C.Senthilkumar et al. [2],In this paper, image compression plays vital role in saving memory storage space and saving time while transmissionimages over network. The color and multispectral image is considered as input image for the image compression. The proposed technique with Enhanced Block Truncation Coding [EBTC] is applied on component of color and multispectral image. The component image is divided into various sub blocks. After evaluating mean values, the number of bits can be reduced by Enhanced Block Truncation Coding. Finally, compression ratio table is generated using the parameters such as MSE, SNR, PSNR, CR, BR and CT. The proposed method is implemented through standard color and multispectral images using MATLAB Version 8.1 R2013a.

Jing-Ming Guo et al. [3], Block truncation committal to writing (BTC) has been thought of extremely economical compression technique for many years. In addition, this strategy can give phenomenal preparing proficiency by 
abusing the nature parallelism preferred standpoint of the spot dispersion, and great picture quality can likewise be offered through co-upgrading the class grid and diffused network of the dab dissemination. As per the test comes about, the proposed DDBTC is better than the previous mistake diffused BTC as far as different target picture quality appraisal strategies and in addition preparing productivity.

An altered Block Truncation Coding utilizing max-min quantizer (MBTC) is proposed in this paper to conquer the previously mentioned disadvantages. In the customary BTC, quantization is done in light of the mean and standard deviation of the pixel esteems in each piece. In the proposed strategy, rather than utilizing the mean and standard deviation, a normal estimation of the most extreme, least and mean of the pieces of pixels is taken as the edge for quantization.

Jayamol Mathews et al. [4], with the emerging multimedia technology, image data has been generated at high volume. It is thus important to reduce the image file sizes for storage and effective communication. Block Truncation Coding (BTC) is a lossy image compression technique which uses moment preserving quantization method for compressing digital gray level images. Even though this method retains the visual quality of the reconstructed image with good compression ratio, it shows some artifacts like staircase effect, raggedness, etc. near the edges. A set of advanced BTC variants reported in literature were studied and it was found that though the compression efficiency is good, the quality of the image has to be improved. A modified Block Truncation Coding using max-min quantizer (MBTC) is proposed in this paper to overcome the above mentioned drawbacks. In the conventional BTC, quantization is done based on the mean and standard deviation of the pixel values in each block. In the proposed method, instead of using the mean and standard deviation, an average value of the maximum, minimum and mean of the blocks of pixels is taken as the threshold for quantization. Experimental analysis shows an improvement in the visual quality of the reconstructed image by reducing the mean square error between the original and the reconstructed image. Since this method involves less number of simple computations, the time taken by this algorithm is also very less when compared with BTC.

Seddeq E. Ghrare et al. [5], with the continuing growth of modern communication technologies, demand for image data compression is increasing rapidly. Techniques for achieving data compression can be divided into two basic approaches: spatial coding and Transform coding. This research paper presents a proposed method for the compression of digital images using hybrid compression method based on Block Truncation Coding (BTC) and Walsh Hadamard Transform (WHT). The objective of this hybrid approach is to achieve higher compression ratio by applying BTC and WHT. Several grayscale test images are used to evaluate the coding efficency and performance of the hybrid method and compared with the BTC and WHT respectively. It is generally shown that the proposed method gives better results.

Ki-Won Oh et al. [6], this paper presents a parallel implementation of hybrid vector quantizer-based block truncation coding using Open Computing Language (OpenCL). Processing dependency in the conventional algorithm is removed by partitioning the input image and modifying neighboring reference pixel configuration. Experimental results show that the parallel implementation drastically reduce processing time by $6 \sim 7$ times with significant visual quality improvement.

\section{IMAGE COMPRESSION}

Image compression algorithms must assume that there exists correlation between neighboring pixel values, called spatial redundancy, and correlation between different color planes (in color images), called spectral redundancy. Video compression algorithms exploit the correlation between adjacent frames in a sequence of images (video), called temporal redundancy. The amount of redundancy in an image depends on the correlation existing among the pixels in the image, which in turn relies on the types of image. There are five types of image:

A bi-level image, pixels in this image can have one of two values so that each pixel is represented by one bit

A grayscale image, a pixel in such an image can have one of the $n$ values 0 through $n-1$, denoting one of $2 n$ shades of gray.

A continuous tone image (natural image), adjacent pixels are very close in this type image so that the degree of correlation presence among the pixels is high. Hence, we may achieve good compression. A pixel in such an image is represented by either a single number (in case of a grayscale image) or by three components (in case of a color image).

A discrete-tone image (graphical image), this is normally an artificial image. Adjacent pixels in a discrete-tone image often are either identical or vary significantly in value. Compression can be achieved by removing redundancy in input image file. Each type of image may have a feature of redundancy, but they may occur indifferent ways. Therefore, there is no compression method that is good for all images. In this thesis, we deal with methods to compress grayscale image because it is the basic image type, All other image types can be illustrated as a grayscale or a set of grayscale images. In principle, the image processing methods designed for grayscale images can be directly applied to color and video images by processing each color plane, or image frames separately.

\section{BLOCK TRUNCATION CODE}

The encoding method of VQ is time consuming, whereas its decoding method uses table look-up method and is very fast. This method results in higher compression ratio, though quality of the reconstructed image is usually not as good as BTC. BTC is a simple and fast method, which enables high quality reconstruction but bit-rate is also high. Comparatively, the encoder of BTC is faster than that of VQ, while its decoder is little slower. A compromise between these two methods gives a fast decoder, maintains good quality for reconstructed image with moderate bit-rate. Again, this hybrid method can also be used in image feature extraction. That means the compressed data due to this method can directly be used to compute image features like, edge [5-6], and so on

The method of selection of the best fit pattern for an image block $\mathrm{B}$ of size $n \times n$ is as follows. For an image block $\mathrm{B}$, let the pixels coordinates are $x_{1}, x_{2}, \ldots \ldots \ldots . x_{n 2}$ and the corresponding pixel intensities are $f\left(x_{i}\right)$. Available patterns are, say, $P_{1}, P_{2} \ldots \ldots \ldots \ldots . . ., P_{M}$ of size $n \times n$ and the levels present in a pattern are represented by $t$ where $1 \leq t \leq Q$. Thus, any pattern is represented as

$$
k m_{1}=k^{\prime}(A-d)+\left(k-k^{\prime}\right)(A+d)
$$




$$
k m_{2}=k^{\prime}(A-d)^{2}+\left(k-k^{\prime}\right)(A+d)^{2}
$$

Solving for $\mathrm{A}$ and $\mathrm{d}$ we get

$$
\begin{aligned}
& A=m_{1}+\frac{\sigma\left(2 k^{\prime}-k\right)}{2 \sqrt{k^{\prime}\left(k-k^{\prime}\right)}} \\
& d=m_{1}+\frac{\sigma k}{2 \sqrt{k^{\prime}\left(k-k^{\prime}\right)}}
\end{aligned}
$$

Hence, intensity $\hat{f}\left(x_{i}\right)$ of the pixels of the corresponding block in the reconstructed image is given

$$
\hat{f}\left(x_{i}\right)=\left\{\begin{array}{l}
A+d \text { if } x_{i} \in C_{1} \\
A-d \text { if } x_{i} \in C_{1}
\end{array}\right.
$$

It is clear that $\mathrm{a}=\mathrm{A}-\mathrm{d}$ and $\mathrm{b}=\mathrm{A}+\mathrm{d}$, where $\mathrm{a}$ and $\mathrm{b}$ are the quantization levels for partition.

A vector quantizer (VQ) is composed of two components: an encoder and a decoder. Both the encoder and the decoder have the same codebook. After the encoder takes an input vector, it makes a full search in the codebook to find the closest matching codeword, with a minimum Euclidean distance to the input vector.

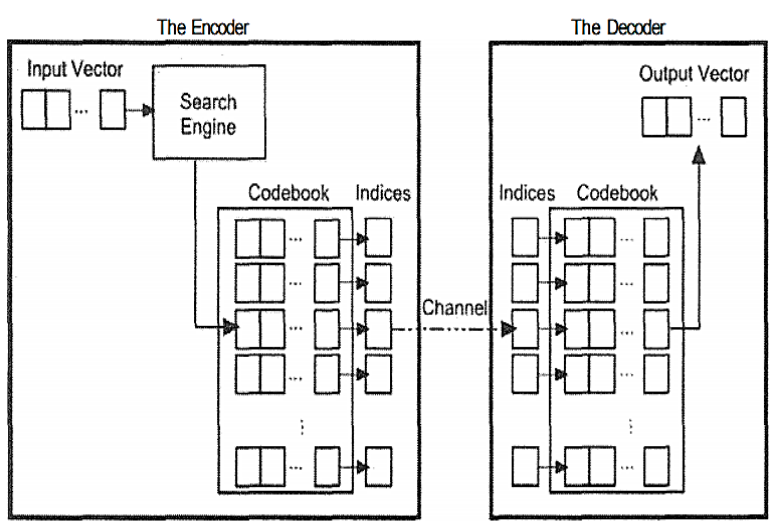

Figure 1: Block diagram of Vector Quantizer

Once the closest codeword is found, the index of that codeword is taken as output for storage or transmission. Since the decoder has the same codebook as the encoder, it is able to look up the codebook for each incoming index and retrieve the corresponding codeword. Compression is achieved because only the index sequence needs to be stored or transmitted. Fig.3.3 shows a block diagram of the encoder and decoder in VQ. For image compression, the input vectors can be formed by dividing the input image into small blocks of the same size pixels $(4 \times 4$ or $8 \times 8)$, with each small block taken as an input vector. The codebook can be generated by means of training with different methods, and the LBG algorithm is one common choice for its easy implementation. The compression performance of a VQ can be measured with Mean Squared Error (MSE), which has been defined in the previous chapter, or Peak Signal to Noise Ratio (PSNR).

Image compression standards like JPEG and JPEG2000 give better reconstructed image quality and compression rate than other compression schemes and these standards are used in almost all the fields. But low computational systems like
System on Chip (SoC) are in need of a low complexity and fast compression schemes for real time applications. BTC is one of the low complexity and fast algorithms for real time applications. Its main attraction is its ease of implementation. But its bit rate is high compared to other compression schemes.

In order to improve the PSNR of BTC images, the best values for the low-mean ' $a$ ' and high-mean ' $b$ ' for the BTC image blocks can be estimated using vector quantization method. A suitable technique has been developed in this research and the procedure is described next, using a $4 \times 4$ block example. The procedure can be used for larger size blocks also, such as $8 \times 8$, $16 \times 16,32$ x 32 etc.

\section{CONCLUSION}

In this survey paper we have shown an image compression method for which the decoder would be very efficient. Such method is suitable in situations where image or image is compressed once but decoded frequently. It is clear that the decoding time due to spatial domain based compression is much less than that of the sub-band compression techniques. Two widely used spatial domain compression techniques are block truncation coding (BTC) and vector quantization (VQ). BTC method results in good quality image with high bit-rate, while the VQ is well known for low bit-rate but produces poor quality images. In this paper the study of different types of technique for image compression. In this paper implementation of the proposed algorithm and results are based on that algorithm is discussed for the different block size i.e. $4 * 4,8 * 8$ and $16 * 16$.

\section{REFERENCES}

[1] Sunwoong Kim and Hyuk-Jae Lee, "RGBW Image Compression by Low-Complexity Adaptive Multi-Level Block Truncation Coding", IEEE Transactions on Consumer Electronics, Vol. 62, No. 4, November 2016.

[2] C. Senthilkumar, "Color and Multispectral Image Compression using Enhanced Block Truncation Coding [E-BTC] Scheme", accepted to be presented at the IEEEWiSPNET, PP. 01-06, 2016 IEEE.

[3] Jing-Ming Guo, Senior Member, IEEE, and Yun-Fu Liu, Member, IEEE, "Improved Block Truncation Coding Using Optimized Dot Diffusion", IEEE Transactions on Image Processing, Vol. 23, No. 3, March 2014.

[4] Jayamol Mathews, Madhu S. Nair, "Modified BTC Algorithm for Gray Scale Images using max-min Quantizer", 978-1-4673-5090-7/13/\$31.00 @2013 IEEE.

[5] M. Brunig and W. Niehsen. Fast full search block matching. IEEE Transactions on Circuits and Systems for Video Technology, 11:241 - 247, 2001.

[6] K. W. Chan and K. L. Chan. Optimisation of multi-level block truncation coding. Signal Processing: Image Communication, 16:445 - 459, 2001.

[7] Ki-Won Oh and Kang-Sun Choi, "Parallel Implementation of Hybrid Vector Quantizerbased Block Truncation Coding for Mobile Display Stream Compression”, IEEE ISCE 20141569954165.

[8] Seddeq E. Ghrare and Ahmed R. Khobaiz, "Digital Image Compression using Block Truncation Coding and Walsh Hadamard Transform Hybrid Technique”, 2014 IEEE 2014 International Conference on Computer, Communication, and Control Technology 
(I4CT 2014), September 2 - 4, 2014 - Langkawi, Kedah, Malaysia.

[9] C. C. Chang and T. S. Chen. New tree-structured vector quantization with closed-coupled multipath searching method. Optical Engineering, 36:1713 -1720, 1997.
[10] C. C. Chang, H. C. Hsia, and T. S. Chen. A progressive image transmission scheme based on block truncation coding. In LNCS Vol 2105, pages 383-397, 2001. 